\title{
The Nearest High-Velocity Stars Revealed by LAMOST Data Release 1
}

\author{
Jing Zhong ${ }^{1}$, Li Chen ${ }^{1}$, Chao Liu $^{2}$, Richard de Grijs ${ }^{3}$, Jinliang Hou ${ }^{1}$, Shiyin Shen ${ }^{1}$, Zhengyi \\ Shao ${ }^{1}$, Jing $\mathrm{Li}^{1}$, Ali $\mathrm{Luo}^{2}$, Jianrong $\mathrm{Shi}^{2}$, Haotong Zhang ${ }^{2}$, Ming Yang ${ }^{2}$, Licai Deng ${ }^{2}$, Ge $\mathrm{Jin}^{5}$, \\ Yong Zhang ${ }^{6}$, Yonghui $\mathrm{Hou}^{6}$, and Zhenchao Zhang ${ }^{6}$
}

\begin{abstract}
We report the discovery of 28 candidate high-velocity stars (HVSs) at heliocentric distances of less than $3 \mathrm{kpc}$, based on the Large Sky Area Multi-Object Fiber Spectroscopic Telescope (LAMOST) Data Release 1. Our sample of HVS candidates covers a much broader color range than the equivalent ranges discussed in previous studies and comprises the first and largest sample of HVSs in the immediate solar neighborhood, at heliocentric distances less than $1-3 \mathrm{kpc}$. The observed as well as the derived parameters for all candidates are sufficiently accurate to allow us to ascertain their nature as genuine HVSs, of which a subset of 12 objects represents the most promising candidates. Our results also highlight the great potential of discovering statistically large numbers of HVSs of different spectral types in LAMOST survey data. This will ultimately enable us to achieve a better understanding of the nature of Galactic HVSs and their ejection mechanisms, and to constrain the structure of the Galaxy.
\end{abstract}

Subject headings: astrometry — Galaxy: halo — Galaxy: structure — stars: kinematics and dynamics — surveys

\section{Introduction}

Hypervelocity stars (HVSs) move sufficiently fast so that they can escape from the Galaxy's gravitational pull. They may have attained their high velocities through three-body interactions, for instance among binary systems in star clusters (e.g., Leonard 1991; Moyano Loyola \& Hurley 2013, and references therein) or with the supermassive black hole in the Galactic Center (GC; e.g., Hills 1988; Yu \& Tremaine 2003; Brown et al. 2005). While this latter formation mechanism is thought to be very promising, other HVS ejection scenarios are possible, such as those involv-

\footnotetext{
${ }^{1}$ Key Laboratory for Research in Galaxies and Cosmology, Shanghai Astronomical Observatory, Chinese Academy of Sciences, 80 Nandan Road, Shanghai 200030, China; jzhong@ shao.ac.cn

${ }^{2}$ Key Laboratory of Optical Astronomy, National Astronomical Observatories, Chinese Academy of Sciences, Datun Road 20A, Beijing 100012, China

${ }^{3}$ Kavli Institute for Astronomy \& Astrophysics and Department of Astronomy, Peking University, Yi He Yuan Lu 5, Hai Dian District, Beijing 100871, China

${ }^{5}$ University of Science and Technology of China, Hefei 230026, China

${ }^{6}$ Nanjing Institute of Astronomical Optics and Technology, National Astronomical Observatories, Chinese Academy of Sciences, Nanjing 210042, China
}

ing close encounters of single stars with binary black holes (Yu \& Tremaine 2003) or the disruption of stellar binaries in the Galactic disk (Wang \& Han 2009; Napiwotzki \& Silva 2012).

Until recently, most confirmed and potential HVSs had been identified as early-type stars located at Galactocentric distances greater than $20 \mathrm{kpc}$. At present, dozens of confirmed HVSs are known in the Milky Way (Brown et al. 2005, 2009, 2012; see also Tillich et al. 2009, 2010), most of which are massive B-type stars. To distinguish among HVS ejection mechanisms and place firm constraints on the origin of their parent population, construction of larger HVS candidate samples spanning a much wider range of spectral types is imperative. Based on Sloan Digital Sky Survey (SDSS) data, Kollmeier et al. (2009, 2010) attempted to find metal-rich, old-population HVS stars (mainly F/G stellar types) that had been ejected from the GC. However, their non-detection of such old-population ejectees only places a limit on the rate of ejection, which suggests that the stellar mass function in the GC may be top- instead of bottomheavy, or alternatively that the supermassive black hole ejection mechanism often involed for the ejection of lower-mass stars is less effective than expected. 
Recently, Li et al. (2012) reported the discovery of 13 F-type HVSs, located at distances ranging from 3 $\mathrm{kpc}$ to $10 \mathrm{kpc}$. Palladino et al. (2014) identified 20 high-velocity G- and K-dwarf candidates, discovered in the Sloan Extension for Galactic Understanding and Exploration (SEGUE) sample, most of which are located far beyond the solar neighborhood (at distances of 3-6 kpc). The first HVS discovered in the Large Sky Area Multi-Object Fiber Spectroscopic Telescope (LAMOST) survey, a B-type star characterized by a galactrocentric radial velocity component of approximately $477 \mathrm{~km} \mathrm{~s}^{-1}$ and a heliocentric distance of $\sim 13$ $\mathrm{kpc}$, was recently reported by Zheng et al. (2014).

Here we report the discovery of 28 solar-neighborhood HVS candidates based on the LAMOST Data Release 1 (DR1). All candidates have velocities, with respect to the Galactic rest frame, in excess of $300 \mathrm{~km} \mathrm{~s}^{-1}$; 12 objects move faster than $400 \mathrm{~km} \mathrm{~s}^{-1}$. Our HVS candidate sample covers a much more extended color range than addressed in previous studies, while most targets are located at heliocentric distances within 3 $\mathrm{kpc}$ (some even within $1 \mathrm{kpc}$ ). Our sample comprises the first large HVS sample in the immediate solar neighborhood. As a consequence, it is of great importance for investigating the various HVS ejection mechanisms, which may be more complex than previously thought (e.g., Palladino et al. 2014).

This Letter is organized as follows. In Section 2 , a brief description of the LAMOST survey data and our candidate selection is presented. We discuss distance estimates to our HVS candidates and derive their kinematic properties in Section 3. The key results are presented in Section 4

\section{LAMOST Survey Data and HVS Candidate Selection}

\subsection{LAMOST DR1 Data}

LAMOST - the "Guoshoujing telescope"-is a Schmidt telescope with an effective aperture of $4 \mathrm{~m}$. It is equipped with 4000 fibers that can be deployed across a $5^{\circ}$ (diameter) field of view, with a spectral resolution of $R \simeq 1800$ over the wavelength range from $3800 \AA$ to $9100 \AA$ Cui et al. 2012; Zhao et al. 2012).

DR1, based on a year of pilot operations and the first season of official survey science, contains $1.7 \times 10^{6}$ spectra suitable for accurate radial-velocity (RV) measurements. Most of these spectra represent observa- tions obtained as part of the LAMOST Experiment for Galactic Understanding and Exploration (LEGUE) survey (Deng et al. 2012) aimed at unveiling the structure of the Milky Way. A subset of some 1.3 million spectra, mostly of bright ( $r<17$ mag) stars spanning a broad color range, are characterized by signal-to-noise ratios $(\mathrm{S} / \mathrm{N})$ greater than 5 in the $\operatorname{SDSS} g, r$, and $i$ filters. The sample selection criteria (cf. Carlin et al. 2012; Chen et al. 2012; Yang et al. 2012; Zhang et al. 2012) favor inclusion of nearby main-sequence stars, simply because of their preponderance along any line of sight.

\subsection{HVS Candidate Selection}

By 2013 March 20, regular LAMOST survey operations had observed and processed around one million stellar spectra. The standard reduction pipeline (Zhao et al. 2012) converts two-dimensional into onedimensional (1D) spectra, applies flat-field corrections, combines the blue and red spectral ranges, and takes care of wavelength calibration and sky subtraction. The pipeline also provides stellar RVs based on cross correlation with the ELODIE library (Prugniel et al. 2007). Although the process of stellar parameter determination, including of the appropriate stellar surface gravities $(\log g)$, effective temperatures $\left(T_{\text {eff }}\right)$, and metallicities $([\mathrm{Fe} / \mathrm{H}])$, is still being refined, stellar RVs based on "good-quality spectra" $(\mathrm{S} / \mathrm{N}>5)$ are highly accurate (cf. Luo et al. 2012, where the quotes refer to verbatim text from that reference), with uncertainties of order $13 \mathrm{~km} \mathrm{~s}^{-1}$.

For a systematic investigation of HVS candidates, we first selected the 14,650 stars in the LAMOST DR1 with absolute RV values greater than $200 \mathrm{~km} \mathrm{~s}^{-1}$. Next, to derive three-dimensional (space) velocities, we identified the 6148 targets for which we had both proper motions - from the Fourth U.S. Naval Observatory CCD Astrograph Catalog (UCAC4) - and SDSS DR9 photometry $(r<18.5 \mathrm{mag})$ available, with sufficient $\mathrm{S} / \mathrm{N}$ in both the $g$ and $i$ bands $(\mathrm{S} / \mathrm{N} \geq 6$ and $\mathrm{S} / \mathrm{N}$ $\geq 20$, respectively). This resulted in an astrometric catalog containing 2600 candidates.

To derive photometric distances, we need reliable estimates of the stellar metallicities. Starting from our 2600 candidate HVSs, we further excluded stars for which either their LAMOST-based $[\mathrm{Fe} / \mathrm{H}]$ values are not available or their SDSS colors fall outside either the $[0.2<(g-r)<0.6,0.8<(u-g)<1.3]$ mag or $[0.1<(r-i)<1.6]$ mag ranges (Ivezić et al. 2008), 


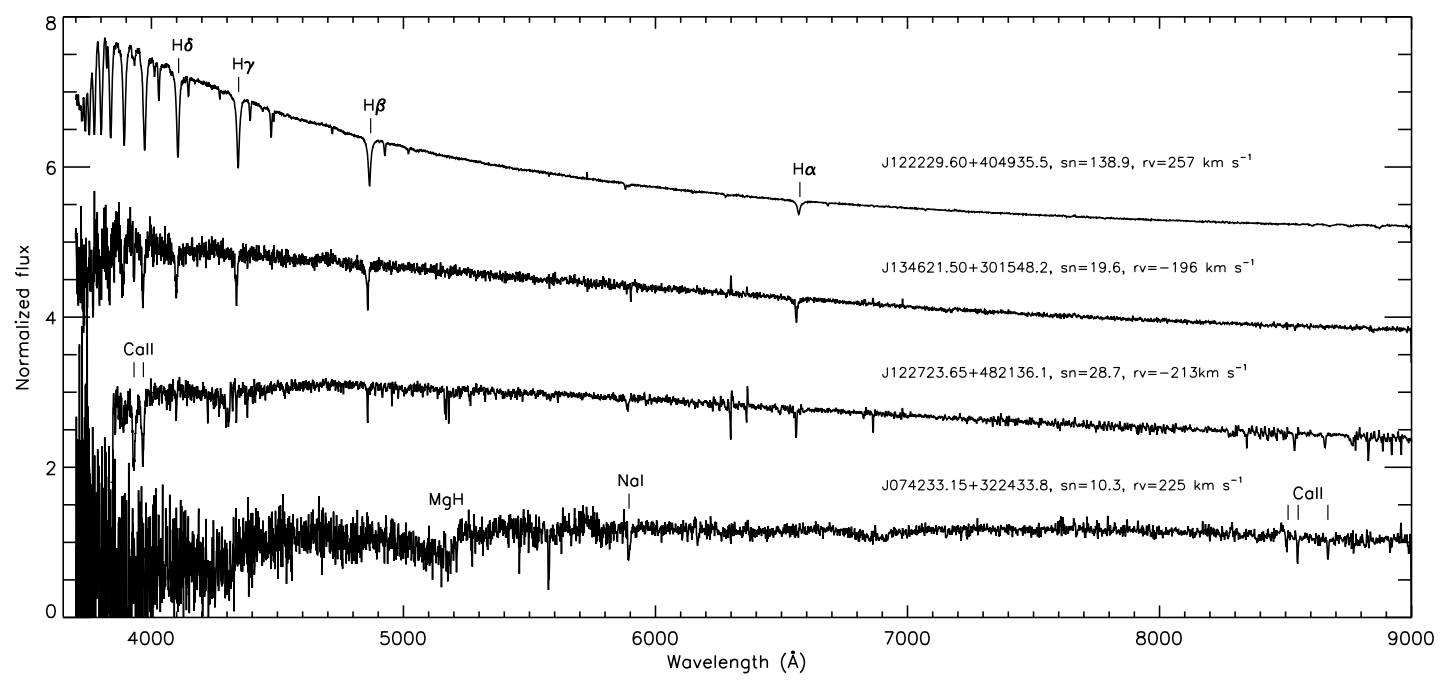

Fig. 1.- Typical spectral sequence representative of our HVS candidates. A number of different absorption lines are used for measuring the average wavelength shift and computing the RVs. The LAMOST designation, $\mathrm{S} / \mathrm{N}$, and RV are shown above the respective spectrum.

where sufficiently accurate photometric metallicities can be derived. We subsequently selected the remaining 1800 candidates to calculate the preliminary stellar distances and space velocities in the Galactic rest frame $\left(V_{\mathrm{gt}}\right)$. Upon imposing an additional selection criterion of $V_{\mathrm{gt}}>300 \mathrm{~km} \mathrm{~s}^{-1}$, only approximately 200 candidates were left. We visually inspected this final subsample and calculated the equivalent widths of the $\operatorname{Mg} b(5180 \AA), \mathrm{H} \beta(4863 \AA)$, and TiO lines $(6150 \AA)$ for each LAMOST spectrum. We next verified the stellar RVs in our sample catalog and excluded those spectra that were characterized by low $\mathrm{S} / \mathrm{N}$. The RVs resulting from such observations were insufficiently reliable because they lacked a sufficient number $(\geq 3)$ of spectral lines for calibration. We also excluded those spectra that more likely represented the characteristics of red giant stars according to the classification criteria derived by Liu et al. (2014). We finally obtained a HVS candidate sample containing 28 objects: see Table 1 .

\section{HVS Candidate Verification}

\subsection{RV Verification}

To verify the LAMOST RV results, we used the IRAF/rv.rvidlines package. Figure 1 shows four typical spectra representative of our sample objects.
For early-type stars (top and second panels), we predominantly used the Balmer lines- $\mathrm{H} \alpha(6563 \AA), \mathrm{H} \beta$, $\mathrm{H} \gamma(4341 \AA)$, and $\mathrm{H} \delta(4102 \AA)$ - to compute the average wavelength shift and obtain the best-fitting RV; for late-type stars (third and bottom panels), we used other appropriate lines for calibration, including the calcium Ca II) H and K lines at, respectively, $3933 \AA$ and 3968 $\AA$ and the near-infrared (near-IR) (Ca II triplet at 8498 $\AA, 8542 \AA$, and $8662 \AA$. The mean RV errors for all spectra were less than $10 \mathrm{~km} \mathrm{~s}^{-1}$.

Among our data set of 28 promising HVSs, five also have SDSS RV measurements. For the latter stars, we found reasonable consistency in the RV results. The residual standard deviation $(\sigma)$ was $16.8 \mathrm{~km} \mathrm{~s}^{-1}$ when comparing our RV results with the SDSS DR9 values, and $\sigma=29.7 \mathrm{~km} \mathrm{~s}^{-1}$ when comparing the LAMOST 1D pipeline results with the SDSS data. This also implies that our IRAF reduction is more reliable than the LAMOST 1D pipeline results. Below (e.g., in Section 4), we compare the properties of our candidate HVSs in the context of literature-based determinations, where available, in more detail.

Note that a well-known, hyper-runaway B-type star (HIP 60350; see also Maitzen et al. 1998; Tenjes et al. 2001; Irrgang et al. 2010), is also included in our initial sample catalog (LAMOST designation: J122229.60+404935.5; see the top panel of Figure 1). This star's RV is $248 \pm 13$ 
$\mathrm{km} \mathrm{s}^{-1}$ based on the LAMOST 1D pipeline and $257 \pm 8$ $\mathrm{km} \mathrm{s}^{-1}$ as derived from our IRAF results. This is in agreement with Irrgang et al. (2010), who found a barycentric RV of $v_{\mathrm{rad}}=262 \pm 5 \mathrm{~km} \mathrm{~s}^{-1}$, based on a high-resolution optical echelle spectrum.

\subsection{Distance Derivation}

To obtain the six-dimensional (6D) phase-space coordinates of the HVS candidates, we first adopted the photometric parallax relations of Ivezić et al. (2008) to calculate the candidates' absolute $r$-band magnitudes, $M_{r}$. For objects with $0.2<(g-i)<4.0 \mathrm{mag}$, we used

$$
M_{r}(g-i,[\mathrm{Fe} / \mathrm{H}])=M_{0}+\Delta M_{r},
$$

where $\Delta M_{r}$ and $M_{0}$ are given by, respectively, Eqs (A2) and (A7) of Ivezić et al. (2008). Where available, we used the $[\mathrm{Fe} / \mathrm{H}]$ values derived by the LAMOST pipeline. For objects without LAMOST metallicity determinations, photometric metallicities were adopted based on Eq. (4) of Ivezić et al. (2008), for $0.2<(g-r)<0.6 \mathrm{mag}$ and $0.8<(u-g)<1.3$ mag. For stars that did not meet either of these color criteria, but which had $(r-i)$ colors in the range $0.1<(r-i)<1.6 \mathrm{mag}$, a "bright" photometric parallax relation based on Eq. (2) of Jurić et al. (2008) was used.

To derive the appropriate extinction corrections, we used the Rayleigh-Jeans color-excess method (Majewski et al. 2011), which estimates reddening values on a star-by-star basis using a combination of near- and mid-IR data from the Two Micron AllSky Survey's (2MASS) Point Source Catalog and the WISE catalog. Specifically, we used data in the $H$ and WISE $W 2(4.6 \mu \mathrm{m})$ bands:

$$
A\left(K_{\mathrm{s}}\right)=0.918\left\{H-[4.6 \mu \mathrm{m}]-(H-[4.6 \mu \mathrm{m}])_{0}\right\} .
$$

We adopted $(H-4.6 \mu \mathrm{m})_{0}=0.05 \mathrm{mag}$ (Zasowski et al. 2013 ) and $E\left(J-K_{\mathrm{s}}\right)=1.5 A_{K_{\mathrm{s}}}$ (Indebetouw et al. 2005).

To avoid reddening overcorrections of halo targets, we used the (Schlegel et al. 1998, hereinafter SFD) reddening maps as upper limit to the reddening toward stars in fields at Galactic latitudes $|b| \geq 16^{\circ}$ (cf. Zasowski et al. 2013), adopting

$$
A_{K_{\mathrm{s}}}=0.302 E(B-V)_{\mathrm{SFD}} \text {, }
$$

as long as the $E\left(J-K_{\mathrm{s}}\right)$ value calculated based on the 2MASS and WISE data was greater than $1.2 \times$ the
SFD-derived value (Zasowski et al. 2013). The visual extinction $A_{V}$ can then be obtained from $A_{K_{\mathrm{s}}} / A_{V}=$ 0.118 (Cardelli et al. 1989). For negative $A_{K_{\mathrm{s}}}$ values, we adopted the reddening values from the integrated SFD maps. We derived the $r$-band extinction, $A_{r}$, using $A_{r} / A_{V}=R_{r} / R_{V}=0.8$, where $R_{r}=2.31$ is given by Yuan et al. (2013) and $R_{V}=3.1$ (Fitzpatrick 1999).

The distances thus derived from the de-reddened $r$ band distance modulus, combined with the positions of our 28 HVS candidates in 6D phase-space, are sufficiently accurate and reliable to support our identification of these objects as genuine HVSs. We illustrated this in Section 3.1. where we discussed the properties of the highest-velocity star in our sample in the context of our full database of observed and derived parameters. In Section 4 we provide a number of examples of candidate stars whose spectra exhibit features as expected from their designations in the literature.

\subsection{Spatial Velocity Calculations}

We converted the RV, distance, and proper motions of each HVS candidate to $V_{x}, V_{y}$, and $V_{z}$ in the (Cartesian) Galactic coordinate system centered on the GC, where the $x$ axis points to the direction opposite to that of the Sun, the $y$ axis is aligned with the direction of Galactic rotation, and the $z$ axis points toward the North Galactic Pole. The Sun is located at $x=-7.8 \mathrm{kpc}$ (McMillan \& Binney 2010). The velocity of the local standard of rest (LSR) is set at $220 \mathrm{~km} \mathrm{~s}^{-1}$, and the motion of the Sun with respect to the LSR is $\left(U_{0}, V_{0}, W_{0}\right)=(11.1,12.24,7.25) \mathrm{km}$ $\mathrm{s}^{-1}$ (Schönrich \& Binney 2009). We used the Markov Chain Monte Carlo technique to extract the $U, V$, and $W$ components of all candidates from the normally distributed parameter space of RV, distance, and proper motions. We estimated the typical uncertainty associated with the RVs based on LAMOST spectra at 13 $\mathrm{km} \mathrm{s}^{-1}$ (Luo et al. 2012). The resulting photometric distance accuracy is then approximately $10 \%$, assuming r.m.s. values of the absolute $M_{r}$ magnitudes of approximately $0.05 \mathrm{mag}$ (Ivezić et al. 2008) and photometric $r$-band magnitude uncertainties of $\sim 0.02 \mathrm{mag}$ (York et al. 2000). The kinematic parameters pertaining to our 28 HVS candidates thus derived are included in Table 2.

\section{Conclusions}

In this Letter, we have reported the discovery of a sample of 28 HVSs selected from the LAMOST DR1, 
Table 1: Basic Parameters of the 28 HVS Candidates.

\begin{tabular}{|c|c|c|c|c|c|c|c|c|c|c|c|c|c|c|c|c|c|}
\hline Designation & $\begin{array}{l}\text { R.A. } \\
\text { deg }\end{array}$ & $\begin{array}{l}\text { Dec. } \\
\text { deg }\end{array}$ & $\begin{array}{l}\mu_{\alpha} \cos (\delta) \\
\operatorname{mas}^{-1} r^{-1}\end{array}$ & $\begin{array}{c}\mu_{\delta} \\
\operatorname{mas} \mathrm{yr}^{-1}\end{array}$ & $\begin{array}{c}u \\
\mathrm{mag}\end{array}$ & $\begin{array}{c}g \\
\text { mag }\end{array}$ & $\begin{array}{c}r \\
\mathrm{mag}\end{array}$ & $\begin{array}{c}i \\
\mathrm{mag}\end{array}$ & $\begin{array}{c}z \\
\mathrm{mag}\end{array}$ & $\begin{array}{c}J \\
\mathrm{mag}\end{array}$ & $\begin{array}{c}H \\
\mathrm{mag}\end{array}$ & $\begin{array}{c}K_{\mathrm{S}} \\
\mathrm{mag}\end{array}$ & $\begin{array}{c}W 2 \\
\mathrm{mag}\end{array}$ & $\begin{array}{r}\mathrm{Fe} / \mathrm{H}] \\
\mathrm{dex}\end{array}$ & $\begin{array}{c}V_{\mathrm{r}}^{a} \\
\mathrm{~km} \mathrm{~s}^{-1}\end{array}$ & $\begin{array}{c}V_{\mathrm{r}}^{b} \\
\mathrm{~km} \mathrm{~s}^{-1}\end{array}$ & $\overline{\text { SpTy }}$ \\
\hline J085819.90+150352.4 & 134.58293 & 15.064578 & $-21.8 \pm 2.6$ & $-54.3 \pm 3.0$ & 15.23 & $\frac{14.22}{14.22}$ & 14.38 & 14.13 & 13.82 & 12.94 & 12.69 & 12.64 & 12.63 & -1.11 & 428 & 415 & $\overline{\mathrm{A} 5}$ \\
\hline J092707.09+242752.9 & 141.77954 & 24.464709 & $-59.8 \pm 8.5$ & $-2.7 \pm 8.0$ & 17.17 & 16.18 & 15.83 & 15.90 & 15.67 & 14.90 & 14.58 & 14.50 & 14.40 & -1.56 & 306 & 308 & F5 \\
\hline $\mathrm{J} 074233.15+322433.8$ & 115.63816 & 32.409389 & $-156.0 \pm 8.0$ & $-353.0 \pm 8.0$ & 19.96 & 17.47 & 16.12 & 15.57 & 15.26 & 14.10 & 13.62 & 13.43 & 13.09 & - & 233 & 225 & K7 \\
\hline $\mathrm{J} 225922.58+262409.9$ & 344.84410 & 26.402775 & $-49.3 \pm 4.2$ & $72.2 \pm 4.5$ & 16.19 & 15.26 & 14.96 & 14.84 & 14.81 & 14.04 & 13.70 & 13.64 & 13.62 & - & -229 & -220 & B6 \\
\hline $\mathrm{J} 115445.93+523520.3$ & 178.69142 & 52.588984 & $56.3 \pm 2.2$ & $-76.4 \pm 2.6$ & 16.42 & 15.36 & 14.95 & 14.90 & 14.75 & 13.88 & 13.55 & 13.53 & 13.46 & - & -228 & -219 & F2 \\
\hline J082932.48+282613.3 & 127.38536 & 28.437039 & $-196.0 \pm 8.0$ & $-395.0 \pm 8.0$ & 18.99 & 16.51 & 15.18 & 14.64 & 14.34 & 13.20 & 12.64 & 12.44 & 12.21 & - & 279 & 282 & K7 \\
\hline $\mathrm{J} 231232.05+265039.5$ & 348.13358 & 26.844326 & $63.6 \pm 2.9$ & $14.6 \pm 3.0$ & 16.12 & 15.08 & 14.63 & 14.78 & 14.37 & 13.48 & 13.08 & 13.00 & 13.01 & $-\overline{0.70}$ & -235 & -196 & F5 \\
\hline $\mathrm{J} 135931.65+413121.6$ & 209.88189 & 41.522689 & $-70.3 \pm 2.8$ & $-78.4 \pm 3.7$ & 16.47 & 15.47 & 15.11 & 14.96 & 14.93 & 14.11 & 13.75 & 13.66 & 13.70 & - & 261 & 244 & F5 \\
\hline $\mathrm{J} 134621.50+301548.2$ & 206.58962 & 30.263401 & $-15.2 \pm 3.9$ & $-58.4 \pm 4.2$ & 16.56 & 15.66 & 15.45 & 15.37 & 15.37 & 14.59 & 14.37 & 14.37 & 14.21 & - & -244 & -196 & A0 \\
\hline $\mathrm{J} 110208.65+575200.2$ & 165.53608 & 57.866742 & $70.6 \pm 1.9$ & $-84.0 \pm 1.6$ & 14.67 & 13.42 & 14.61 & 12.95 & 13.24 & 11.96 & 11.65 & 11.63 & 11.61 & -1.16 & -219 & -227 & A0 \\
\hline $\mathrm{J} 224145.15+292428.4$ & 340.43813 & 29.407892 & $-15.3 \pm 7.2$ & $-60.4 \pm 10.0$ & 16.03 & 15.09 & 14.88 & 14.78 & 14.76 & 13.99 & 13.72 & 13.72 & 13.73 & - & -220 & -210 & A0 \\
\hline $\mathrm{J} 124020.02+453259.6$ & 190.08345 & 45.549896 & $26.2 \pm 2.6$ & $-52.8 \pm 2.6$ & 16.32 & 15.42 & 15.15 & 15.05 & 15.06 & 14.34 & 14.01 & 14.02 & 13.95 & -1.30 & -219 & -195 & A0 \\
\hline J123134.97+424736.1 & 187.89571 & 42.793379 & $17.2 \pm 2.9$ & $-49.2 \pm 2.6$ & 16.83 & 15.89 & 15.57 & 15.46 & 15.44 & 14.59 & 14.31 & 14.18 & 14.36 & -1.35 & -254 & -266 & Fo \\
\hline $\mathrm{J} 094122.37-000822.2$ & 145.34323 & -0.139505 & $-46.5 \pm 4.4$ & $-67.6 \pm 4.6$ & 16.54 & 15.54 & 15.23 & 15.07 & 15.05 & 14.20 & 14.01 & 13.91 & 13.85 & -1.62 & 430 & 426 & Fo \\
\hline $\mathrm{J} 073050.71+293212.6$ & 112.71130 & 29.536856 & $21.9 \pm 3.2$ & $-77.7 \pm 2.9$ & 15.85 & 14.86 & 14.52 & 14.40 & 14.37 & 13.56 & 13.21 & 13.22 & 13.16 & -1.20 & -309 & -291 & F5 \\
\hline $\mathrm{J} 123641.48+443715.9$ & 189.17285 & 44.621092 & $-23.7 \pm 3.2$ & $-55.6 \pm 3.0$ & 17.22 & 16.35 & 16.07 & 15.95 & 15.95 & 15.12 & 14.90 & 14.95 & 14.79 & -1.79 & -257 & -259 & Fo \\
\hline $\mathrm{J} 085623.74+211322.3$ & 134.09893 & 21.222872 & $-60.0 \pm 3.4$ & $-47.3 \pm 2.6$ & 15.09 & 14.15 & 13.81 & 13.88 & 13.77 & 12.83 & 12.50 & 12.51 & 12.48 & -1.46 & 223 & 235 & Fo \\
\hline $\mathrm{J} 173650.63+060814.4$ & 264.21098 & 6.1373510 & $-30.9 \pm 18.7$ & $-15.5 \pm 7.0$ & 16.57 & 15.25 & 14.73 & 14.50 & 14.39 & 13.44 & 13.03 & 12.99 & 12.95 & -1.51 & -436 & -416 & F5 \\
\hline $\mathrm{J} 224207.91+072808.3$ & 340.53297 & 7.4689990 & $60.2 \pm 2.3$ & $-42.7 \pm 2.6$ & 16.06 & 15.28 & 16.33 & 14.60 & 14.43 & 13.50 & 13.18 & 13.24 & 13.15 & -2.18 & -462 & -436 & A0 \\
\hline J091849.92-005331.5 & 139.70801 & -0.892091 & $-45.3 \pm 3.2$ & $-85.7 \pm 3.0$ & 14.79 & 13.91 & 13.73 & 13.47 & 13.42 & 12.48 & 12.24 & 12.20 & 12.15 & -1.81 & 453 & 465 & A5 \\
\hline $\mathrm{J} 122723.65+482136.1$ & 186.84858 & 48.360029 & $15.3 \pm 3.5$ & $-88.2 \pm 3.7$ & 17.04 & 15.88 & 15.44 & 15.28 & 15.19 & 14.36 & 13.96 & 13.84 & 13.89 & -1.11 & -209 & -213 & G0 \\
\hline $\mathrm{J} 124330.95+423119.9$ & 190.87898 & 42.522216 & $-25.4 \pm 2.5$ & $-55.5 \pm 2.5$ & 16.74 & 15.90 & 15.64 & 15.56 & 15.53 & 14.85 & 14.56 & 14.46 & 14.55 & -2.20 & -229 & -263 & A0 \\
\hline $\mathrm{J} 121437.93+542522.9$ & 183.65808 & 54.423052 & $17.0 \pm 3.8$ & $-62.6 \pm 4.0$ & 15.93 & 14.98 & 14.72 & 14.63 & 14.65 & 13.82 & 13.59 & 13.54 & 13.57 & - & -203 & -197 & A0 \\
\hline $\mathrm{J} 212028.30-013323.4$ & 320.11794 & -1.556502 & $51.2 \pm 3.8$ & $-47.0 \pm 4.0$ & 16.87 & 15.99 & 15.67 & 15.54 & 15.53 & 14.76 & 14.36 & 14.54 & 14.30 & -2.18 & -338 & -311 & Fo \\
\hline J080608.76+063349.8 & 121.53652 & 6.5638360 & $14.2 \pm 4.6$ & $-20.4 \pm 5.1$ & 16.84 & 15.91 & 15.65 & 15.57 & 15.54 & 14.84 & 14.50 & 14.43 & 14.64 & -1.25 & 427 & 423 & A0 \\
\hline J065436.50+170313.8 & 103.64375 & 17.053856 & $-1.4 \pm 2.1$ & $-16.0 \pm 2.3$ & 15.57 & 14.05 & 13.55 & 13.40 & 13.60 & 12.31 & 11.98 & 11.91 & 11.84 & -1.23 & 290 & 307 & F9 \\
\hline $\mathrm{J} 102757.74+090030.0$ & 156.99059 & 9.008337 & $-160.0 \pm 8.0$ & $-35.0 \pm 8.0$ & 15.67 & 14.50 & 14.03 & 14.06 & 13.73 & 12.28 & 12.29 & 12.80 & 12.38 & - & 293 & 314 & $\mathrm{G} 2$ \\
\hline $\mathrm{J} 120758.23+093231.9$ & 181.99265 & 9.542214 & $-100.0 \pm 8.0$ & $-137.0 \pm 8.0$ & 15.83 & 14.60 & 14.00 & 14.24 & 13.65 & 12.04 & 12.07 & 12.65 & 12.10 & -1.55 & 215 & 214 & G2 \\
\hline
\end{tabular}

1D pipeline, while $V_{\mathrm{r}}^{b}$ is based on our IRAF reduction; SpTy is the spectral type from the LAMOST 1D pipeline.

Table 2: Spatial Positions and Kinematic Parameters of the 28 HVS Candidates.

\begin{tabular}{|c|c|c|c|c|c|c|c|c|c|c|c|c|c|}
\hline Name & Designation & $\begin{array}{l}\text { R.A. } \\
\text { deg }\end{array}$ & $\begin{array}{l}\text { Dec. } \\
\text { deg }\end{array}$ & $\begin{array}{c}A_{V} \\
\text { mag }\end{array}$ & $\begin{array}{l}D_{\odot} \\
\text { kpc }\end{array}$ & $\begin{array}{l}r_{\mathrm{gcc}} \\
\mathrm{kpc}\end{array}$ & $\begin{array}{c}x \\
\mathrm{kpc}\end{array}$ & $\begin{array}{c}y \\
\mathrm{kpc}\end{array}$ & $\begin{array}{c}z \\
\mathrm{kpc}\end{array}$ & $\begin{array}{c}V_{x} \\
\mathrm{~km} \mathrm{~s}^{-1}\end{array}$ & $\begin{array}{c}V_{y} \\
\mathrm{~km} \mathrm{~s}^{-1}\end{array}$ & $\begin{array}{c}V_{z} \\
\mathrm{~km} \mathrm{~s}^{-1}\end{array}$ & $\begin{array}{c}V_{\mathrm{gt}} \\
\mathrm{km} \mathrm{s}^{-1}\end{array}$ \\
\hline LMST_HVS1 & J085819.90+150352.4 & 134.58293 & 15.064578 & 0.062 & 3.3 & 10.5 & -10.3 & -1.5 & 1.9 & $-227 \pm 7$ & $-705 \pm 42$ & $-288 \pm 6$ & $795 \pm 35$ \\
\hline LMST_HVS2 & $\mathrm{J} 092707.09+242752.9$ & 141.77954 & 24.464709 & 0.085 & 2.3 & 9.6 & -9.5 & -0.6 & 1.6 & $-655 \pm 10$ & $92 \pm 79$ & $-233 \pm 45$ & $707 \pm 7$ \\
\hline LMST_HVS3 & $\mathrm{J} 074233.15+322433.8$ & 115.63816 & 32.409389 & 0.104 & 0.4 & 8.4 & -8.3 & -0.0 & 0.1 & $-329 \pm 18$ & $-412 \pm 51$ & $-382 \pm 24$ & $652 \pm 56$ \\
\hline LMST_HVS4 & $\mathrm{J} 225922.58+262409.9$ & 344.84410 & 26.402775 & 0.256 & 0.9 & 8.1 & -8.0 & 0.8 & -0.5 & $46 \pm 26$ & $248 \pm 34$ & $470 \pm 36$ & $535 \pm 46$ \\
\hline LMST_HVS5 & $\mathrm{J} 115445.93+523520.3$ & 178.69142 & 52.588984 & 0.051 & 1.1 & 8.4 & -8.4 & 0.3 & 1.0 & $492 \pm 41$ & $-50 \pm 2$ & $48 \pm 31$ & $497 \pm 43$ \\
\hline LMST_HVS6 & $\mathrm{J} 082932.48+282613.3$ & 127.38536 & 28.437039 & 0.085 & 0.2 & 8.2 & -8.2 & -0.0 & 0.1 & $-311 \pm 15$ & $-318 \pm 42$ & $-177 \pm 15$ & $480 \pm 43$ \\
\hline LMST_HVS7 & $\mathrm{J} 231232.05+265039.5$ & 348.13358 & 26.844326 & 0.171 & 1.6 & 8.3 & -8.1 & 1.4 & -0.8 & $-465 \pm 82$ & $-55 \pm 0$ & $10 \pm 6$ & $469 \pm 81$ \\
\hline LMST_HVS8 & $\mathrm{J} 135931.65+413121.6$ & 209.88189 & 41.522689 & 0.033 & 1.0 & 8.0 & -7.9 & 0.3 & 0.9 & $31 \pm 0$ & $-164 \pm 22$ & $409 \pm 22$ & $443 \pm 28$ \\
\hline LMST_HVS9 & $\mathrm{J} 134621.50+301548.2$ & 206.58962 & 30.263401 & 0.045 & 2.0 & 7.9 & -7.7 & 0.3 & 2.0 & $238 \pm 29$ & $-326 \pm 8$ & $-129 \pm 8$ & $425 \pm 11$ \\
\hline LMST_HVS10 & $\mathrm{J} 110208.65+575200.2$ & 165.53608 & 57.866742 & 0.024 & 0.8 & 8.4 & -8.4 & 0.2 & 0.6 & $409 \pm 28$ & $-56 \pm 9$ & $85 \pm 37$ & $423 \pm 35$ \\
\hline LMST_HVS11 & $\mathrm{J} 224145.15+292428.4$ & 340.43813 & 29.407892 & 0.192 & 1.4 & 8.1 & -8.0 & 1.3 & -0.6 & $326 \pm 49$ & $-76 \pm 11$ & $-171 \pm 5$ & $377 \pm 43$ \\
\hline LMST_HVS12 & $\mathrm{J} 124020.02+453259.6$ & 190.08345 & 45.549896 & 0.042 & 1.4 & 8.4 & -8.2 & 0.3 & 1.3 & $373 \pm 36$ & $-10 \pm 7$ & $-58 \pm 19$ & $379 \pm 32$ \\
\hline LMST_HVS13 & $\mathrm{J} 123134.97+424736.1$ & 187.89571 & 42.793379 & 0.073 & 1.3 & 8.3 & -8.2 & 0.2 & 1.3 & $316 \pm 30$ & $-32 \pm 6$ & $-152 \pm 18$ & $354 \pm 18$ \\
\hline LMST_HVS14 & $\mathrm{J} 094122.37-000822.2$ & 145.34323 & -0.139505 & 0.167 & 0.9 & 8.4 & -8.4 & -0.6 & 0.5 & $-189 \pm 2$ & $-291 \pm 16$ & $-10 \pm 4$ & $348 \pm 14$ \\
\hline LMST_HVS15 & $\mathrm{J} 073050.71+293212.6$ & 112.71130 & 29.536856 & 0.189 & 0.8 & 8.7 & -8.7 & -0.1 & 0.2 & $321 \pm 3$ & $-24 \pm 25$ & $-117 \pm 16$ & $344 \pm 7$ \\
\hline LMST_HVS16 & $\mathrm{J} 123641.48+443715.9$ & 189.17285 & 44.621092 & 0.045 & 1.7 & 8.5 & -8.3 & 0.3 & 1.6 & $116 \pm 13$ & $-294 \pm 11$ & $-116 \pm 18$ & $337 \pm 7$ \\
\hline LMST_HVS17 & $\mathrm{J} 085623.74+211322.3$ & 134.09893 & 21.222872 & 0.078 & 1.0 & 8.7 & -8.7 & -0.3 & 0.6 & $-292 \pm 15$ & $-50 \pm 13$ & $-132 \pm 5$ & $325 \pm 17$ \\
\hline LMST_HVS18 & $\mathrm{J} 173650.63+060814.4$ & 264.21098 & 6.1373510 & 0.217 & 0.4 & 7.6 & -7.6 & 0.2 & 0.1 & $-313 \pm 5$ & $-17 \pm 32$ & $-92 \pm 19$ & $329 \pm 5$ \\
\hline LMST_HVS19 & J224207.91+072808.3 & 340.53297 & 7.4689990 & 0.316 & 0.8 & 7.9 & -7.8 & 0.6 & -0.5 & $-184 \pm 23$ & $-253 \pm 4$ & $92 \pm 29$ & $329 \pm 8$ \\
\hline LMST_HVS20 & J091849.92-005331.5 & 139.70801 & -0.892091 & 0.077 & 0.5 & 8.2 & -8.2 & -0.3 & 0.2 & $-202 \pm 2$ & $-239 \pm 18$ & $76 \pm 2$ & $322 \pm 14$ \\
\hline LMST_HVS21 & $\mathrm{J} 122723.65+482136.1$ & 186.84858 & 48.360029 & 0.033 & 0.9 & 8.2 & -8.2 & 0.2 & 0.8 & $298 \pm 26$ & $-113 \pm 5$ & $-43 \pm 22$ & $323 \pm 22$ \\
\hline LMST_HVS22 & $\mathrm{J} 124330.95+423119.9$ & 190.87898 & 42.522216 & 0.077 & 1.5 & 8.4 & -8.2 & 0.3 & 1.5 & $103 \pm 9$ & $-260 \pm 16$ & $-142 \pm 18$ & $315 \pm 7$ \\
\hline LMST_HVS23 & $\mathrm{J} 121437.93+542522.9$ & 183.65808 & 54.423052 & 0.051 & 1.2 & 8.4 & -8.4 & 0.4 & 1.1 & $302 \pm 29$ & $-85 \pm 9$ & $14 \pm 22$ & $316 \pm 26$ \\
\hline LMST_HVS24 & J212028.30-013323.4 & 320.11794 & -1.556502 & 0.085 & 1.1 & 7.4 & -7.4 & 0.7 & -0.6 & $-231 \pm 25$ & $-164 \pm 4$ & $-125 \pm 42$ & $312 \pm 33$ \\
\hline LMST_HVS25 & $\mathrm{J} 080608.76+063349.8$ & 121.53652 & 6.5638360 & 0.077 & 1.9 & 9.5 & -9.5 & -1.0 & 0.6 & $-170 \pm 6$ & $-176 \pm 7$ & $185 \pm 64$ & $312 \pm 33$ \\
\hline LMST_HVS26 & J065436.50+170313.8 & 103.64375 & 17.053856 & 0.700 & 0.2 & 8.2 & -8.2 & -0.0 & 0.0 & $-273 \pm 11$ & $120 \pm 4$ & $41 \pm 4$ & $302 \pm 9$ \\
\hline LMST_HVS27 & $\mathrm{J} 102757.74+090030.0$ & 156.99059 & 9.008337 & 0.090 & 0.7 & 8.3 & -8.2 & -0.3 & 0.6 & $-525 \pm 35$ & $-154 \pm 4$ & $-89 \pm 3$ & $555 \pm 34$ \\
\hline LMST_HVS28 & $\mathrm{J} 120758.23+093231.9$ & 181.99265 & 9.542214 & 0.050 & 0.8 & 8.0 & -8.0 & -0.2 & 0.7 & $-62 \pm 4$ & $-443 \pm 26$ & $-15 \pm 4$ & $447 \pm 25$ \\
\hline
\end{tabular}


Table 3: Distances and Derived Kinematic Parameters of the 28 HVS Candidates from the Literature.

\begin{tabular}{|c|c|c|c|c|c|c|c|c|}
\hline Name & $\begin{array}{l}\text { R.A. } \\
\text { deg }\end{array}$ & $\begin{array}{l}\begin{array}{l}\text { Dec. } \\
\text { deg }\end{array} \\
\end{array}$ & $\begin{array}{l}D_{\odot} \\
\mathrm{kpc}\end{array}$ & $\begin{array}{l}V_{x} \\
\mathrm{~km} \mathrm{~s}^{-1}\end{array}$ & $\begin{array}{l}V_{y} \\
\mathrm{~km} \mathrm{~s}^{-1}\end{array}$ & $\begin{array}{l}V_{z} \\
\mathrm{~km} \mathrm{~s}^{-1}\end{array}$ & $\begin{array}{l}V_{\mathrm{gt}} \\
\mathrm{km} \mathrm{s}^{-1} \\
\end{array}$ & Ref. \\
\hline LMST_HVS1 & 134.58293 & 15.064578 & 1462 & -254 & -283 & -9 & 381 & 1 \\
\hline LMST_HVS2 & 141.77954 & 24.464709 & - & - & - & - & - & - \\
\hline LMST_HVS3 & 115.63816 & 32.409389 & - & - & - & - & - & - \\
\hline LMST_HVS4 & 344.84410 & 26.402775 & 1329 & 57 & 319 & 592 & 675 & 1 \\
\hline LMST_HVS5 & 178.69142 & 52.588984 & 1010 & 444 & -26 & 21 & 445 & 1 \\
\hline LMST_HVS6 & 127.38536 & 28.437039 & - & - & - & - & - & - \\
\hline LMST_HVS7 & 348.13358 & 26.844326 & - & - & - & - & - & - \\
\hline LMST_HVS8 & 209.88189 & 41.522689 & $1890(714)$ & $40(28)$ & $-571(-19)$ & 557 (357) & $799(359)$ & $1(2)$ \\
\hline LMST_HVS9 & 206.58962 & 30.263401 & 2475 & 287 & -436 & -117 & 536 & 1 \\
\hline LMST_HVS10 & 165.53608 & 57.866742 & 130 & 167 & 126 & -135 & 250 & 1 \\
\hline LMST_HVS11 & 340.43813 & 29.407892 & - & - & - & - & - & - \\
\hline LMST_HVS12 & 190.08345 & 45.549896 & 2468 & 611 & -158 & -30 & 632 & 1 \\
\hline LMST_HVS13 & 187.89571 & 42.793379 & 2378 & 493 & -185 & -84 & 534 & 1 \\
\hline LMST_HVS14 & 145.34323 & -0.139505 & $1484(1267)$ & $-195(-193)$ & $-431(-375)$ & $-169(-106)$ & $503(435)$ & 1(2) \\
\hline LMST_HVS15 & 112.71130 & 29.536856 & 798 & 321 & -24 & -118 & 343 & 2 \\
\hline LMST_HVS16 & 189.17285 & 44.621092 & - & - & - & - & - & - \\
\hline LMST_HVS17 & 134.09893 & 21.222872 & 1031 & -293 & -52 & -135 & 327 & 1 \\
\hline LMST_HVS18 & 264.21098 & 6.1373510 & - & - & - & - & - & - \\
\hline LMST_HVS19 & 340.53297 & 7.4689990 & 3658 & -565 & -830 & -597 & 1169 & 1 \\
\hline LMST_HVS 20 & 139.70801 & -0.892091 & $1455(596)$ & $-157(-199)$ & $-523(-263)$ & $-244(48)$ & 599(333) & $1(2)$ \\
\hline LMST_HVS21 & 186.84858 & 48.360029 & 5099 & 1322 & -1394 & 608 & 2015 & 1 \\
\hline LMST_HVS22 & 190.87898 & 42.522216 & 2422 & 129 & -500 & -86 & 524 & 1 \\
\hline LMST_HVS23 & 183.65808 & 54.423052 & 1204 & 292 & -78 & 7 & 303 & 1 \\
\hline LMST_HVS24 & 320.11794 & -1.556502 & - & & - & - & - & - \\
\hline LMST_HVS25 & 121.53652 & 6.5638360 & 2415 & -138 & -220 & 187 & 320 & 1 \\
\hline LMST_HVS26 & 103.64375 & 17.053856 & - & - & - & - & - & - \\
\hline LMST_HVS27 & 156.99059 & 9.008337 & 1878 & -1143 & -494 & -594 & 1380 & 1 \\
\hline LMST_HVS28 & 181.99265 & 9.542214 & 1698 & -147 & -1113 & -263 & 1153 & 1 \\
\hline
\end{tabular}

of which 12 objects are the most likely HVS candidates. Our sample of HVS candidates covers a much broader color range than the equivalent ranges discussed in previous studies and comprises the first and largest sample of HVSs in the solar neighborhood.

We have access to sufficiently accurate observed and derived parameters for all 28 HVS candidates to ascertain their nature as genuine HVSs. To further verify the reliability of the sample of $28 \mathrm{HVSs}$, we matched our candidates with other catalogs. We first checked their proper motions based on the PPMXL catalog (Roeser et al. 2010) and did not find any significant differences between the PPMXL and UCAC4 catalogs for these $28 \mathrm{HVSs}$. Two candidates in the New Luyten Two Tenths (NLTT) catalog (Luyten 1979a,b), LMST_HVS3 and LMST_HVS6, have proper motions of $\left[\mu_{\alpha} \cos (\delta), \mu_{\delta}\right]=(-154.2,-359.1)$ mas $\mathrm{yr}^{-1}$ and $(-197.2,-384.9)$ mas $\mathrm{yr}^{-1}$, respectively (Salim \& Gould 2003). LMST_HVS3 is identified as a K-type star in the NLTT catalog, where it is catalogued as object 309-34; careful analysis of our LAMOST spectra also classifies it as a K-dwarf star. (The nearest NLTT star, is located at a projected distance of approximately $12^{\prime}$, so that there can be no confusion as to our candidate HVS's nature on this basis.) We have double checked the spectral features of all 28 HVS candidates, none of which exhibit characteristics of white dwarfs or binary systems. LMST_HVS1 is also listed as a high proper-motion star $\left[\mu_{\alpha} \cos (\delta)=-20.8\right.$ mas $\mathrm{yr}^{-1}, \mu_{\delta}=-54.8$ mas yr$\left.^{-1}\right]$ in the catalog of stars with high proper motions (Ivanov 2008). For all three stars, the similarity of the proper motions indicates the reliability of our kinematic data.

Since the uncertainty in distance is the major contributor to the uncertainties in the derived total (space) velocities, we use additional available photometric distances from Pickles \& Depagne (2010) and Gontcharov et al. (2011) to recalculate the total velocities of our sample of $28 \mathrm{HVSs}$. Based on these literature-based distances, the derived total velocities and $(U, V, W)$ components in the Galactic rest frame are listed in Table 3, while the proper motions and RVs used are included in Table 1 The space velocities of the 28 candidates included in Table 3 suggest that the candidates we discovered are highly probable HVSs because of their intrinsically large proper motions and RVs.

The results reported here also highlight the great potential of discovering statistically large numbers of HVSs of different spectral types in LAMOST survey data. We will continue to perform further systematic HVS searches based on the LAMOST data; the resulting stellar samples will eventually enable us to better understand the nature of the HVSs themselves and ultimately constrain the structure of the Galaxy.

This work was supported by "973 Program” 2014 CB845702, the Strategic Priority Research Program "The Emergence of Cosmological Structures" of the Chinese Academy of Sciences (CAS; grant XDB09010100) and by the National Natural Science Foundation of 
China (NSFC) under grants 11373054, 11073038, 11073001, and 11373010. The Guoshoujing Telescope (LAMOST) is a National Major Scientific Project built by the CAS. Funding for the project has been provided by the National Development and Reform Commission. LAMOST is operated and managed by the National Astronomical Observatories, CAS.

\section{REFERENCES}

Brown, W. R., Geller, M. J., Kenyon, S. J., \& Kurtz, M. J. 2005, ApJ, 622, L33

Brown, W. R., Geller, M. J., \& Kenyon, S. J. 2009, ApJ, 690, 1639

Brown, W. R., Geller, M. J., \& Kenyon, S. J. 2012, ApJ, 751, 55

Cardelli, J. A., Clayton, G. C., \& Mathis, J. S. 1989, ApJ, 345, 245

Carlin, J. L., Lépine, S., Newberg, H. J., et al. 2012, Res. Astron. Astrophys., 12, 755

Chen, L., Hou, J.-L., Yu, J.-C., et al. 2012, Res. Astron. Astrophys., 12, 805

Cui, X., Zhao, Y., Chu, Y., Li, G., Li, Q., Zhang, L., Su, H., et al. 2012, Res. Astron. Astrophys., 12, 1197

Deng, L. C., Newberg, H. J., Liu, C., et al. 2012, Res. Astron. Astrophys., 12, 735

Fitzpatrick, E. L. 1999, PASP, 111, 63

Gontcharov, G. A., Bajkova, A. T., Fedorov, P. N., \& Akhmetov, V. S. 2011, MNRAS, 413, 158

Hills, J. G. 1988, Natur, 331, 687

Indebetouw, R., Mathis, J. S., Babler, B. L., et al. 2005, ApJ, 619, 931

Irrgang, A., Przybilla, N., Heber, U., Nieva, M. F., \& Schuh, S. 2010, ApJ, 711, 138

Ivanov, G. A. 2008, Kinematika i Fizika Nebesnykh Tel, 24, 480

Ivezić, Ž., Sesar, B., Jurić, M., et al. 2008, ApJ, 684, 287

Jurić, M., Ivezić, Ž., Brooks, A., et al. 2008, ApJ, 673, 864
Kollmeier, J. A., Gould, A., Knapp, G., \& Beers, T. C. 2009, ApJ, 697, 1543

Kollmeier, J. A., Gould, A., Rockosi, C., et al. 2010, ApJ, 723, 812

Leonard, P. J. T. 1991, AJ, 101, 562

Li, Y., Luo, A., Zhao, G., et al. 2012, ApJ, 744, L24

Liu, C., Deng, L. C., Carlin, J. L., et al. 2014, ApJ, submitted (arXiv:1404.4871)

Luo, A., Zhang, H., Zhao, Y., Zhao, G., Cui, X., Li, G., Chu, Y., et al. 2012, Res. Astron. Astrophys., 12, 1243

Luyten, W. J. 1979a, New Luyten Catalogue of stars with proper motions larger than two tenths of an arcsecond, vol. 1: +90 degrees to +30 degrees.

Luyten, W. J. 1979b, New Luyten Catalogue of stars with proper motions larger than two tenths of an arcsecond, vol. 2: +30 degrees to 0 degrees.

Maitzen, H. M., Paunzen, E., Pressberger, R., Slettebak, A., \& Wagner, R. M. 1998, A\&A, 339, 782

Majewski, S. R., Zasowski, G., \& Nidever, D. L. 2011, ApJ, 739, 25

McMillan, P. J., \& Binney, J. J. 2010, MNRAS, 402, 934

Moyano Loyola G. R. I., \& Hurley, J. R. 2013, MNRAS, 434, 2509

Napiwotzki, R., \& Silva, M. D. V. 2012, Mem. Soc. Astron. Italiana, 83, 272

Palladino, L. E., Schlesinger, K. J., HolleyBockelmann, K., Prieto, C. A., Beers, T. C., Lee, Y. S., \& Schneider, D. P. 2014, ApJ, 780, 7

Pickles, A., \& Depagne, É. 2010, PASP, 122, 1437

Prugniel, Ph., Soubiran, C., Koleva, M., \& Le Borgne, D. 2007, ELODIE Library v. 3.1 (arXiv:astro-ph/0703658)

Roeser, S., Demleitner, M., \& Schilbach, E. 2010, AJ, 139,2440

Salim, S., \& Gould, A. 2003, ApJ, 582, 1011

Schlegel, D. J., Finkbeiner, D. P., \& Davis, M. 1998, ApJ, 500, 525 
Schönrich, R., \& Binney, J. 2009, MNRAS, 396, 203

Spagna, A., Lattanzi, M. G., Re Fiorentin, P., \& Smart, R. L. 2010, A\&A, 510, L4

Tenjes, P., Einasto, J., Maitzen, H. M., \& Zinnecker, H. 2001, A\&A, 369, 530

Tillich, A., Przybilla, N., Scholz, R.-D., \& Heber, U. 2009, A\&A, 507, L37

Tillich, A., Przybilla, N., Scholz, R. D., \& Heber, U. 2010, A\&A, 517, A36

Wang, B., \& Han, Z. 2009, A\&A, 508, L27

Yang, F., Carlin, J. L., Liu, C., et al. 2012, Res. Astron. Astrophys., 12, 781

Yuan, H. B., Liu, X. W., \& Xiang, M. S. 2013, MNRAS, 430, 2188

Yu, Q., \& Tremaine, S. 2003, ApJ, 599, 1129

Zacharias, N., Finch, C. T., Girard, T. M., et al. 2013, AJ, 145, 44

Zasowski, G., Johnson, J. A., Frinchaboy, P. M., Majewski, S. R., et al. 2013, AJ, 146, 81

Zhang, Y.-Y., Carlin, J. L., Yang, F., et al. 2012, Res. Astron. Astrophys., 12, 792

Zhao, G., Zhao, Y.-H., Chu, Y.-Q., Jing, Y.-P., \& Deng, L.-C. 2012, Res. Astron. Astrophys., 12, 723

Zheng, Z., Carlin, J. L., Beers, T. C., et al. 2014, ApJ, 785, L23

York, D. G., Adelman, J., Anderson, J. E., Jr., et al. 2000, AJ, 120, 1579

This 2-column preprint was prepared with the AAS ${ }^{\mathrm{A}} \mathrm{T} \mathrm{E}$ X macros v5.2. 